\title{
Philonsorbonne
}

6 | 2012

Année 201 1-2012

\section{Doctorales I}

Résumés des interventions des premières Doctorales de Philosophie (2011)

\section{(2) OpenEdition}

\section{Journals}

Édition électronique

URL : https://journals.openedition.org/philonsorbonne/399

DOI : 10.4000/philonsorbonne.399

ISSN : 2270-7336

Éditeur

Publications de la Sorbonne

\section{Édition imprimée}

Date de publication : 15 juillet 2012

Pagination : $97-125$

ISBN : 978-2-85944-710-6

ISSN : 1255-183X

\section{Référence électronique}

"Doctorales | », Philonsorbonne [En ligne], 6 | 2012, mis en ligne le 04 février 2013, consulté le 10 juin 2021. URL : http://journals.openedition.org/philonsorbonne/399 ; DOI : https://doi.org/10.4000/

philonsorbonne.399

(C) Tous droits réservés 


\section{DOCTORALES I ${ }^{1}$ \\ Année 2010-2011}

Vincent ARDOUREL

Laboratoire de rattachement : IHPST

Thèse dirigée par Jacques Dubucs et Anouk Barberousse vincent.ardourel@gmail.com

\section{Les sciences peuvent-elles se passer des nombres?}

Une des questions les plus discutées en philosophie des mathématiques concerne le statut des nombres. Pour le réalisme ontologique - ou platonisme -, les nombres existent indépendamment de nous. Cette thèse est soutenue par un argument en particulier, celui de l'indispensabilité des nombres en sciences. Il consiste à justifier notre engagement ontologique vis-à-vis des nombres à partir de la constatation selon laquelle ceux-ci s'avèrent indispensables aux sciences empiriques. Certains antiréalistes s'attaquent à cet argument en cherchant à montrer que malgré les apparences, les sciences empiriques peuvent se passer des nombres. Au premier rang figure H. Field qui, dans Science without numbers, met en évidence la possibilité de reformuler la physique sans utiliser les nombres. Je propose ici de discuter la méthodologie adoptée par H. Field, appliquée non pas aux nombres en général, mais à un certain type de nombres seulement, les nombres irrationnels. Ces derniers sont des nombres particulièrement problématiques: la connaissance de leur valeur numérique exacte nous est inaccessible. Elle impliquerait en effet de notre part une capacité de calcul infinie.

Dans cette intervention, je discute un argument, emprunté à R. Carnap, en faveur du caractère non indispensable des nombres irrationnels en

1. Dans cette rubrique sont publiés les résumés des interventions ayant eu lieu lors des premières Doctorales de Philosophie organisées à la Sorbonne, les 20, 21, 27 et 28 mai, par les doctorants Élodie Baget, Élodie Djordjevic, Aurélie KnÜFER, Raphaël Koster, Sophie LaVeran, Alberto NAIBo, et Esther Rogan. 
physique. Dans les Fondements philosophiques de la physique, celui-ci distingue deux types de nombres intervenant en physique, les résultats d'un calcul théorique et les résultats d'une mesure empirique, et il montre que les nombres irrationnels sont toujours du premier type et jamais du second. Dans ces conditions, la question de l'indispensabilité des nombres irrationnels en physique se réduit à celle de l'indispensabilité des calculs théoriques utilisant les nombres irrationnels. Carnap reconnaît la commodité des calculs en physique et par conséquent celle des nombres irrationnels. Il suggère cependant que bien que plus commodes, les nombres irrationnels pourraient ne pas être indispensables en physique. Dans cette intervention, je discute cette conjecture et précise les conditions pour lesquelles la physique peut se passer des nombres irrationnels: la physique peut se passer des nombres irrationnels si et seulement si toutes les déductions physiques établies à l'aide de calculs faisant intervenir des nombres irrationnels peuvent aussi être établies, certes moins commodément, à l'aide de calculs ne faisant intervenir que des nombres rationnels. 
Benjamin BERGER

Laboratoire de rattachement : PhiCo Thèse dirigée par : Renaud Barbaras benjamin.berger@yahoo.fr

\section{Le problème de la méthodologie chez Ruyer}

Ruyer, dans un texte précoce, revendique l'héritage de la "grande voie naturelle de la philosophie », laquelle consiste à «tirer de l'intuition psychologique ce qui peut servir de modèle et d'échantillon à une ontologie $»$. L'anthropomorphisme méthodologique qui en découle roule sur la neutralisation de la singularité ontologique de l'homme. Pour le montrer, nous avons exhumé la préférence que, dans son anthropologie, Ruyer témoigne pour certains des accents du discours matérialiste - par ailleurs disqualifié au plan de l'ontologie. Parce qu'il nous enjoint à remettre l'homme à sa place dans l'univers, le matérialisme s'impose à Ruyer comme une «saine barbarie de la pensée $»^{2}$. Or, que Ruyer salue le nivellement qu'implique le matérialisme renvoie, à bien y penser, à quelque chose de beaucoup plus profond. Le rôle critique qu'il lui concède est, croyons-nous, l'index d'une dette, jamais thématisée, de son panpsychisme et de la décision méthodologique qui le commande, envers le monisme mécaniste dont, jadis, lui-même s'était fait le champion ${ }^{3}$. Notre hypothèse est la suivante : le passage du panmécanisme au panpsychisme procède d'une logique interne au premier; parce qu'au monisme mécaniste se dérobe la différence ontologique propre au moment anthropologique, la censure infligée à

\footnotetext{
1. R. Ruyer, «Le Versant réel du fonctionnement », Revue philosophique de la France et de l'étranger, 1935, p. 346.

2. R. Ruyer, «Ce qui est vivant et ce qui est mort dans le matérialisme », Revue philosophique de la France et de l'étranger, 1933, p. 33. Cf. aussi du même auteur, "Les Idéologies de notre temps et la toile de fond de la science », Les Études philosophiques, 1959, p. 148 .

3. Cf. R. Ruyer, Esquisse d'une philosophie de la structure, Paris, Félix Alcan, 1930.
} 
l'anthropomorphisme méthodologique, de fait, est annulée ${ }^{4}$, de sorte que le panmécanisme, s'il est conséquent, progressivement s'inverse en sa figure opposée. Aussi n'est-ce pas contre, mais bien à partir de sa philosophie première qu'il faut comprendre la reprise de la tradition panpsychiste chez Ruyer, seul, dès lors, à avoir jamais rejoint la « grande voie naturelle de la philosophie » en partant pour ainsi dire du pôle opposé.

4. Voir ce qu'avance Hans Jonas à propos du destin de l'ontologie positiviste, cf. Le Phénomène de la vie, trad. D. Lories, Bruxelles, De Boeck, 2001, p. 49. 
Sébastien BROCA

Laboratoire de rattachement : CETCOPRA

Directeur de thèse : Philippe Breton

E-mail : brocasebastien@yahoo.fr

\section{L'utopie du logiciel libre}

La communication aborde le mouvement pour le logiciel et la culture « libres », à travers une réflexion sur la notion d'utopie.

Les années 1990 semblent avoir été celles du discrédit porté sur l'utopie, ramenée aussi bien à une impossibilité de fait qu'à une dangerosité de droit. Le pathétique écroulement du bloc communiste et le triomphe du capitalisme néolibéral avaient profondément déconsidéré tout au-delà de la société existante. Il était admis que nous étions « condamnés à vivre dans le monde où nous vivons » (François Furet), voire que nous touchions à la «fin de l'histoire » (Francis Fukuyama). L'utopie se réduisait dès lors à la quête dérisoire de rêveurs inconséquents, ou de révolutionnaires patibulaires. Le contexte historique et intellectuel semble aujourd'hui quelque peu différent. À la faveur des multiples crises contemporaines, il redevient possible de penser d'autres devenirs. On peut dès lors revenir à l'utopie, et essayer de dépasser les reproches d'irréalisme ou de (proto) totalitarisme qui lui sont d'ordinaires associés. La philosophie de l'espérance d'Ernst Bloch et notamment son approche de l' «utopie concrète »- fournit quelques arguments en ce sens, en donnant à l'utopie un ancrage ontologique dans l'élément du non-encore-être (Noch-Nicht-Seins).

Cette réhabilitation du concept d'utopie permet de penser un certain renouveau de l'imaginaire politique contemporain, via l'émergence de mouvements en rupture avec les significations centrales de la «rationalité néolibérale » (Pierre Dardot, Christian Laval). L'un de ces mouvements est celui pour le logiciel et la culture «libres ». Il s'est peu à peu détaché du milieu socio-culturel bien particulier qui l'a vu naître - le milieu hacker - et de l'objet qui lui avait donné son impulsion initiale - le logiciel -, pour 
gagner d'autres domaines et séduire d'autres publics. En trente ans, il a produit des réalisations d'envergure (GNU/Linux, Wikipédia), des outils juridiques innovants (licences GPL et Creative Commons), et un discours militant sur la nécessité de promouvoir les biens communs (commons) numériques. Il porte ainsi la vision d'une société autre, fondée sur la collaboration, la circulation des savoirs, et de nouvelles formes de régulations économiques, juridiques et politiques.

Cette utopie, malgré ses faiblesses et ses points aveugles, donne ainsi à penser que, tout compte fait, nous ne sommes peut-être pas condamnés à vivre dans le monde social dans lequel nous vivons. 
Filip BUYSE

Laboratoire de rattachement : CHSPM Thèse dirigée par Chantal Jaquet filip.buyse1@telenet.be

\section{La controverse entre Boyle et Spinoza sur la « rédintégration » du salpêtre}

Spinoza n'a jamais écrit directement de lettre à Boyle, tout comme Boyle n'en a jamais écrit à Spinoza. Néanmoins, il y a des lettres connues dans la littérature sous le nom de correspondance entre Boyle et Spinoza.

En règle générale, tous les commentateurs de cette «correspondance » commencent leur discussion par la visite de Henry Oldenburg à Spinoza. En effet, l'Allemand rend visite à Spinoza pendant l'été 1661, à l'occasion d'un voyage dans son pays natal. De retour à Londres, il envoie une lettre à Spinoza pour l'inviter à maintenir le contact avec lui. C'est le début d'une longue correspondance. Très vite, il envoie à Spinoza un livre de Boyle, The Physiological Essays, en traduction latine, en lui demandant de commenter surtout les expériences faites par Boyle. Dans sa longue réponse, le philosophe hollandais s'excuse de ne pas avoir lu intégralement ce livre mais explique qu'il a pu quand même en lire de façon critique la deuxième partie, celle qui concernait les expériences. Il s'agissait de deux essais : un essai sur le salpêtre et un essai sur la fluidité et la solidité. Dans les lettres 6, 11,13 et 16 (1661-1663), Spinoza entretient une discussion en latin avec Boyle, bien que toujours indirecte, par l'intermédiaire d'Oldenburg.

Dans mes recherches au contraire, je n'ai pas examiné la correspondance à partir de Spinoza, mais plutôt à partir de Boyle. À mon avis, cet examen nous aide à une meilleure compréhension non seulement de l'histoire de la correspondance, mais aussi de son contenu.

Premièrement, cette approche montre clairement que les acteurs majeurs (P. Serrarius, R. Glauber, R. Jones, R. Boyle, Lady Ranelagh, etc.) de la correspondance ne sont pas des membres de la Royal Society, mais plutôt du cercle de Hartlib. 
Deuxièmement, cette approche révèle que la critique menée par Spinoza de l'interprétation de la rédintégration est plutôt une critique adressée au chimiste et alchimiste Glauber qu'à Boyle lui-même, puisque c'est l'Allemand qui a décrit cette réaction chimique pour la première fois. D'après les recherches récentes de W. Newman, Boyle connaissait bien le travail de Glauber sur le salpêtre puisque son ami, le hartlibien B. Worsely, avait visité en 1648-49, l'impressionnant laboratoire de Glauber, qui se trouvait près de la maison natale de Spinoza à Amsterdam; Worsely publiera suite à cela un livre sur le salpêtre, De nitro theses quaedam.

D'après M. Hunter, De Nitro est probablement - aux yeux de Boyle lui-même - l'un des plus importants textes que l'expérimentateur irlandais a écrits. Néanmoins, ce qui importe à Boyle n'est pas la réaction en ellemême, mais plutôt la rédintégration en tant que moyen de défendre sa philosophie mécaniste comme alternative à la philosophie péripatéticienne qu'il réfute catégoriquement.

Dans sa préface, Boyle donne une première définition de la corpuscular philosophy - appellation qu'il préférait à celle de Mechanical Philosophy. C'est dans le contexte de la promotion de cette nouvelle philosophie qu'il faut comprendre De Nitro, et que nous avons relu la correspondance entre Boyle et Spinoza. 
Pierre FASULA

Laboratoire de rattachement : PhiCo (équipe EXeCO)

Directrice de thèse : Christiane Chauviré.

Mail : pierre.fasula@free.fr

Lien : http://execo.univ-paris1.fr/spip.php?article183

\section{Conditionnel contrefactuel ou supposition : la présence du possible chez Musil et Wittgenstein.}

Dans L'Homme sans qualités, Musil définit ainsi l'utopie: «[...] l'utopie est une expérience dans laquelle on observe la modification possible d'un élément et les conséquences que cette modification entraînerait dans ce phénomène complexe que nous appelons la vie $»^{1}$.

Cette définition est tout aussi problématique que ce dont elle est tirée, à savoir la définition, par Mach, de l'expérimentation mentale. Si l'on suit les analyses de Wittgenstein sur ce point, décrire une utopie ou une expérimentation mentale, ce n'est pas faire une expérience d'une manière particulière (en pensée) et obtenir des résultats, mais imaginer faire une expérience et imaginer ses résultats. Une différence importante réside notamment dans le fait que, dans une expérience réelle, les conséquences d'une modification sont observées, alors que, dans une utopie ou une expérimentation mentale, une fois la modification imaginée, il nous faut encore imaginer, développer ses conséquences.

C'est pour cette raison que Wittgenstein voit dans l'utopie et l'expérimentation mentale un type de raisonnement qui est analogue à un calcul. La supposition qui en est le point de départ peut être comparée au «début d'un calcul ${ }^{2}$ de ses conséquences possibles. Ainsi, le développement d'une supposition se distingue à la fois de l'énoncé d'une prédiction et de la formulation d'une norme: si " "il en sera ainsi" sélectionne une possibilité parmi d'autres [et que] "il doit en être ainsi" ne

1. R. Musil, L'Homme sans qualités, Paris, Le Seuil, 1956, tr. P. Jaccottet, t. 1, p. 311.

2. L. Wittgenstein, Dictées de Wittgenstein à Waismann et pour Schlick, Paris, PUF, 1997, tr. C. Chauviré et alii, p. 194. 
voit qu'une seule possibilité $»^{3}$, "supposons que..." est le point de départ de l'imagination et du développement d'une possibilité.

Si l'on revient alors à l'utopie, on peut dire qu'elle n'est ni une prédiction empirique portant sur ce qui est, ni une norme affirmant ce qui doit être, mais le développement d'une possibilité. La conséquence, c'est qu'il n'est donc pas légitime d'en faire soit une rêverie que l'on critiquerait parce que sans lien avec la réalité, soit une norme dangereuse, responsable des atrocités du $\mathrm{XX}^{\mathrm{e}}$ siècle. Pour comprendre la fonction des possibilités utopiques, il peut être intéressant à nouveau de se référer à Wittgenstein et à ce qu'il dit de l'usage des jeux de langage : «Nous construisons le cas fictif particulier d'un jeu, dans le but d'examiner ce qui se passe réellement sous le point de vue de l'analogie avec cet autre jeu»". De même, on pourrait dire que l'utopie est la construction d'un point de comparaison qui permet de dissiper des confusions, de critiquer certaines représentations ou encore de mettre en évidence certains aspects de notre vie. La différence résiderait toutefois dans le fait que, là où les constructions du philosophe sont censées disparaître une fois les concepts clarifiés et la réalité perçue correctement, les utopies sont destinées aussi à enrichir notre vie de possibilités nouvelles.

3. L. Wittgenstein, Remarques sur les fondements des mathématiques, Paris, Gallimard, 1983, tr. M.-A. Lescourret, p. 207.

4. L. Wittgenstein, Dictées de Wittgenstein à Waismann et pour Schlick, op. cit., p. 138. 
Florent FRANCHETTE

Laboratoire de rattachement : IHPST Thèse dirigée par : Jacques Dubucs et Anouk Barberousse florent.franchette@gmail.com

\section{L'hyper-calcul et les paradoxes logiques de l'infini}

Le but de ma présentation a été d'exposer l'un des problèmes que soulève l'hyper-calcul, une notion allant au-delà de la notion traditionnelle de calcul proposée par les logiciens des années 1930. Son intérêt principal réside dans la possibilité de résoudre des problèmes fondamentaux en logique et en physique. Toutefois, une des particularités de l'hyper-calcul est d'autoriser l'utilisation de processus infinis exécutés dans un temps fini. Cette utilisation de l'infini permet aux adversaires de l'hyper-calcul d'invoquer certains paradoxes logiques afin de montrer la contradiction de cette notion. Au cours de mon exposé, j'ai tenté de défendre que de tels paradoxes ne remettaient pas en cause l'hyper-calcul de façon explicite.

Le premier paradoxe fut introduit par Zénon et prend la forme d'une course entre Achille et une tortue. D'après ce paradoxe, Achille ne peut atteindre son point d'arrivée, une tortue, car il est obligé de parcourir la somme d'une infinité d'étapes. La résolution standard du problème de Zénon est de considérer cette somme infinie comme une série mathématique afin de calculer sa limite et d'en déduire qu'elle est finie. Nous pouvons ainsi conclure qu'Achille atteindra bien la tortue en parcourant une distance finie. Il peut être néanmoins objecté que la résolution est une pétition de principe, puisque calculer la somme infinie de la série semble présupposer que l'on puisse effectuer un nombre infini d'opérations en un temps fini. De plus, on peut reprocher à cette résolution de déduire des énoncés empiriques à partir de simples définitions sur les séries mathématiques.

Le second paradoxe a été présenté par James Thomson en 1954. Ce dernier considère une lampe pouvant être éteinte ou allumée et pose ensuite la question suivante : quel est l'état de la lampe si l'on appuie sur son 
interrupteur une infinité de fois ? Pour Thomson, puisque la lampe ne peut être ni éteinte ni allumée à la fin de l'expérience, exécuter un nombre infini d'étapes en un temps fini est contradictoire. Contre ce dernier argument, j'ai défendu une possible solution qui fut apportée par Paul Benacerraf en 1962 : même si la description de Thomson nous renseigne sur l'état de la lampe au cours de l'expérience, elle ne nous renseigne en rien quant à son état à la fin de l'expérience. De ce point de vue, la description est incomplète et nous ne pouvons pas conclure que l'expérience est contradictoire. 
Refik GÜREMEN

Laboratoire de rattachement : GRAMATA. Thèse dirigée par Michel Narcy (CNRS) refikg2001@yahoo.com

\section{Remarques sur l'interprétation de l'animal politique aristotélicien}

Dans les Politiques I, 1, après avoir démontré que la polis fait partie des choses naturelles et que l'homme est un animal politique par nature, Aristote affirme, en 1253a 7-9, qu'il est un animal politique plus (mallon) que n'importe quel animal grégaire. Traditionnellement, on comprend cette comparaison comme l'expression d'une différence de nature entre l'homme et les autres animaux dits «politiques»: seul l'homme serait proprement politique, et cela en vertu de sa possession de la polis, tandis que les autres animaux ne le seraient que dans un sens métaphorique.

Contre cette lecture, Jean-Louis Labarrière, tenant compte du fondement biologique de l'argument d'Aristote, s'interroge sur la possibilité de trouver un sens non métaphorique à cette comparaison. À cette fin, il établit un argument dont le cœur s'établit autour des fragments B82-85 (Düring) du Protreptique. De la même façon que, par une comparaison entre l'homme éveillé et l'homme endormi, Aristote fait une distinction entre le sens plus propre et le sens moins propre du « vivre » humain, Labarrière suggère par une analogie de comprendre que c'est dans un sens souverain que l'homme accomplit la capacité politique : grâce à ses caractéristiques humaines, c'est lui qui exerce au mieux cette capacité, tandis que les autres animaux n'y ont qu'un accès élémentaire, sans pour autant que cet accès soit métaphorique, dans la mesure où ils travaillent et vivent également ensemble ${ }^{1}$.

Pourtant, l'ensemble de l'argument constitué par ces fragments repose sur une comparaison entre deux actualisations d'une même potentialité,

1. Langage, vie politique et mouvement des animaux. Etudes Aristotéliciennes, Paris, Vrin, 2004, p. 99-127. 
et Aristote vise par cet argument à montrer la supériorité de celui qui accomplit un ergon selon sa propre excellence : l'enjeu est plutôt ici la supériorité selon la vertu. Cela dit, l'analogie avec le cas des animaux politiques ne tiendra pas, à moins de supposer une identité générique entre l'œuvre politique de l'homme (la polis) et celle des autres animaux et, ensuite, de faire une comparaison entre eux selon un seul et même critère d'excellence : l'homme serait donc plus politique car il dépasserait les autres animaux selon la vertu politique! Malgré son ingéniosité, il nous semble que l'analyse de Labarrière ne tient pas. 
Gladys KostYRKA

Laboratoire de rattachement : IHPST Thèse dirigée par : Michel Morange et Jean Gayon Gladys.kostyrka@wanadoo.fr

\section{Classer les virus, classer les vivants}

La biologie appréhende son objet à l'aide d'un important travail de classification. Les « entités biologiques » sont ainsi séparées en organiques / non organiques; en pathogènes / non pathogènes, en bactéries/archées, mais aussi en vivantes / non vivantes (par exemple, les virus sont des entités «biologiques » considérées comme non vivantes).

Les virus sont généralement considérés comme faisant partie des « microbes». Pourtant, loin d'être classés - comme le sont les bactéries, autres microbes célèbres -, avec les vivants, les virus font l'objet d'une classification distincte, qui a ses propres règles. On observe ainsi deux classifications qui ne se croisent pas: l'arbre du vivant d'une part (eucaryotes, bactéries, archées) reflète l'évolution des vivants; les classifications virales d'autre part (selon la parenté notamment) reflètent l'évolution des virus.

Cette séparation est remise en cause par la mise en évidence d'un important «transfert horizontal» de gènes entre bactéries et virus, et entre archées et virus, qui suggère que les virus influent de manière significative sur l'évolution des vivants, et inversement. Mais si tel est le cas, alors ne devient-il pas nécessaire de former une classification qui reflète la coévolution des virus et des vivants?

Sur ce point, les biologistes s'opposent. Pour les uns, les virus ne sont pas vivants, donc ils ne peuvent être classés avec les vivants. Pour les autres, les virus sont vivants, donc ils doivent être classés avec les vivants. Pour d'autres encore, la seule chose qui importe est qu'il y ait co-évolution : peu importe que les virus soient ou non vivants, il faut les classer aussi avec les 
vivants. Cette dernière position soutient que la nature des virus n'a aucune importance au vu du problème envisagé (qu'est-ce qui évolue ? comment dresser une classification capable de rendre compte de cette évolution ?). Elle dissocie ainsi le problème de la définition (des virus, des vivants) et celui de leur classification.

Notre recherche cherche à répondre à deux questions :

- L'opposition entre ces différents biologistes tient-elle au seul problème de la définition du vivant (et de la nature des virus) ? Ou reposet-elle aussi sur la fonction qu'on assigne aux classifications?

- Peut-on classer sans définir? Plus généralement, quel rapport existet-il entre classification et définition? 
Sophie LEFEEZ

Laboratoire de rattachement : CETCOPRA Thèse dirigée par M. Gérard Dubey, et financée par EADS IW Sophie.Lefeez@malix.univ-paris1.fr

\section{La complexité des matériels militaires et les représentations de « faire la guerre »}

Les récents conflits ont montré que posséder des matériels high-tech n'assure pas de victoire rapide et durable sur le terrain, même face à un adversaire inférieur techniquement. Quelles sont donc les représentations de la façon de faire la guerre qui poussent à rechercher la supériorité technique aujourd'hui dans l'armée française, et comment les ingénieurs et les opérationnels se positionnent-ils par rapport à cette complexité ?

La forte complexité technique des matériels ne permet plus une maîtrise complète du fonctionnement de l'objet technique, tant pour les industriels concepteurs que pour la Direction Générale de l'Armement (DGA), chargée du suivi des programmes d'armement. Dès lors, on peut s'interroger sur la capacité de la DGA à maintenir une expertise technique lorsque la connaissance n'est plus que théorique et partielle. En outre, pousser toujours plus loin la complexité technique quand la maîtrise de l'ensemble du système n'est plus permise devient problématique.

Pourtant, la complexification se poursuit. Une raison se trouve dans les fondements de la science moderne: la vision mécaniste de l'univers développée par les pères fondateurs a donné naissance à un monde perçu comme prédictible. Et si l'on peut prévoir l'avenir, n'a-t-on pas le devoir de s'y préparer au mieux ? Cette croyance alimente une optimisation technique des matériels perçue comme étant indispensable pour se préparer au mieux aux conflits futurs. Or, la guerre réellement menée ne peut correspondre à celle imaginée, l'adversaire n'ayant aucun intérêt à nous faire la guerre à laquelle nous nous sommes préparés. 
En outre, l'accent mis sur le rôle de la technique questionne la place laissée à l'Homme dans le combat. En effet, l'optimisation technique a tendance à engendrer des objets fermés au sens de Simondon, alors même que leur longue durée de vie et l'imprévisibilité de la guerre nécessiterait au contraire de les faire très ouverts afin de faciliter les détournements d'usage.

Deux regards s'opposent ici : par leur formation et leur fonction, les ingénieurs (concepteurs) ont une vision plutôt technocentrée tandis que les opérationnels (usagers) sont davantage anthropocentrés. De plus, la plupart des ingénieurs conçoivent ces objets techniques avec une faible connaissance de leur contexte d'emploi, une lacune perçue par les usagers.

Les choix techniques reflètent un processus d'acquisition où chaque groupe d'acteurs agit selon ses intérêts, ses contraintes et ses croyances. 
Baptiste MONSAINGEON

Laboratoire de rattachement : CETCOPRA Thèse dirigée par Alain Gras et Sophie Poirot-Delpech baptiste.monsaingeon@malix.univ-paris1.fr

\section{Le souci du tri. Des pratiques domestiques de mise au rebut.}

Emblèmes de la négativité, les déchets font aujourd'hui l'objet d'un consensus. Le trop plein détritique semble avoir contraint pouvoirs publics et «jeteurs » en tous genres à agir avec raison: il n'est plus possible de remplir des trous, il n'y aura bientôt plus de place. Ce presque rien, à qui Socrate refusait déjà dans le Parménide toute idéalité, est donc passé d'un oubli caractéristique à une prise en charge systématique, sur le mode impératif : «pour sauver la planète, trions nos déchets !» En une vingtaine d'années, la gestion des déchets ménagers a donc acquis le statut «d'enjeu environnemental ». Au quotidien, ce lien admis entre déchets et action de protection de l'environnement se concrétise autour de la pratique du tri sélectif, érigée en véritable norme sociale.

Au cours de cette communication, il s'est agi de décrire la coexistence, au sein des sociétés occidentales, de deux paradigmes, de deux formes divergentes d'un souci, souvent associé au geste de catégorisation des matières déchues.

Avec le paradigme dit du tri sélectif, il apparaît que ce souci s'assimile avant tout à une forme d'inquiétude liée à la préservation tant de l'espace de vie immédiat du jeteur qu'à celle de son environnement élargi ( «la nature », « la planète »). Ici, trier revient avant tout à répondre à des normes morales, appliquer des consignes qui semblent déterminées par une forme d'interdit de souillure. Le geste est routinier et le déchet reste très largement impensé. À l'inverse, l'étude des pratiques de lombricompostage révèle qu'ici c'est la matière déchue elle-même qui structure le geste de tri. Parce que nourriture 
des lombrics et fertilisant en devenir, les déchets organiques ne sont plus abordés sur le simple mode de leur élimination. Le souci que semble générer ce type de pratiques s'assimile davantage à une forme de soin, d'attention, qu'à la seule inquiétude de «bien faire».

Par opposition à un tri sélectif vécu sur le mode du respect de prescriptions fondées sur une représentation construite de la nature, le lombricompostage est décrit par ses adeptes comme une forme d'expérimentation, de «bidouillage» quotidien avec le vivant. Dans ce paradigme, loin du souci quelque peu figé de préservation d'un « environnement » mal défini, l'attention portée à l'endroit même du déchet impose ici une réévaluation perpétuelle des rapports que nous entretenons avec cela même qui nous environne, offrant ainsi à ce geste banal une fonction heuristique inattendue. 
Camilla RocCA

Équipe d'accueil : Philosophies Contemporaines (EA 3562) - EXeCO

Titre de la thèse : Le sujet en mouvement.

Patočka et le problème de la corrélation.

Thèse dirigée par : Renaud Barbaras et Luca Vanzago

roccamilla@gmail.com

\section{La manifestation : une réalisation dynamique subjective et mondaine}

En concevant une «épochè sans réduction» qui s'étende jusqu'au champ de la conscience transcendantale, Patočka fonde la «phénoménologie asubjective » qui veut remonter à l'apparaitre comme tel, sans l'expliquer en recourant aux lois de l'apparaissant. Privé de son rôle d'« origine constituante »du monde, le sujet est alors simplement un étant apparaissant à l'intérieur du champ phénoménal, chose parmi les choses. Cependant, il est une chose singulière, parce que par lui les autres étants se manifestent : l'être sort de son retrait par l'intermédiaire du sujet, mais indépendamment de son initiative, en tant que le sujet réalise les possibilités que le monde lui offre. En ce sens, on peut attribuer au champ phénoménal une certaine subjectivité, qui a affaire avec son destinataire et non pas avec son constituant. La sphère phénoménale est alors un projet de l'étant en totalité qui englobe également l'ego sum en tant que centre qui se rapporte à soimême à travers tout le reste.

L'accomplissement des possibilités du monde passe par l'action corporelle du sujet dans le monde, par laquelle il réalise dans le même temps soi-même. La corporéité subjective se définit alors existentiellement comme la première des possibilités de l'homme en tant que possibilité de semouvoir : le mouvement est le sens d'être du sujet, et de ce fait l'homme est à même de comprendre le mouvement de tous les autres êtres et de les recueillir en lui. C'est donc le mouvement de notre vie qui porte les choses à leur manifestation. 
Le mouvement de l'existence, au sens de la réalisation de soi, passe par l'enracinement du sujet dans une communauté qui l'accueille et l'accepte, par la lutte pour la conservation de soi à travers le travail et la reproduction, et enfin par la reconnaissance de sa propre finitude et la problématisation du sens de l'être. Ce questionnement philosophique de l'être propre et des choses conduit le Dasein à effectuer un "pas en arrière ", en «transcendant » les données immédiates (y compris soi-même), et donc à s'ouvrir à la transcendance, au fondement de l'apparaître, de l'être qui est un rien, qui se voile en se dévoilant, et qui est présent sous le mode de l'absence. Donc, parce qu'il fait «exploser» la subjectivité égologique husserlienne fermée dans son absoluité et parce que le sujet est, de ce fait, seulement le destinataire du monde situé dans le monde même, Patočka peut remonter à l'auto-manifestation du monde à partir de son propre fond obscur asubjectif. 
Équipe d'accueil : Philosophies Contemporaines (EA 3562) - ExeCO

Titre de la thèse : La transcendance comme problème phénoménologique. Lecture de Merleau-Ponty et Patočka.

Thèse dirigée par : Renaud Barbaras

emresan@gmail.com

\section{La totalité comme promesse}

Nous nous proposons ici de mettre en lumière la spécificité du concept de transcendance en tant que «concept opératoire » qui court à travers tous les textes de Patočka sans faire l'objet d'une réflexion spécifique, mais qui joue néanmoins un rôle décisif au regard de la question de l'apparaitre. En fait, Patočka propose une alternative à la dimension transcendantale de la perspective husserlienne d'une "transcendance dans l'immanence», élaborée sur le chemin cartésien et reposant sur la primauté de l'immanence sur la transcendance.

Face à l'ampleur du sujet, dans cette intervention nous nous contenterons seulement de faire une synthèse du sens de la transcendance entre description phénoménologique et description métaphysique. La méthode intuitive de la phénoménologie husserlienne exclut toute aventure métaphysique, tout excès spéculatif. Aux yeux de Patočka, une telle approche correspond à un oubli ou à un refoulement de la métaphysique, alors qu'il fait partie de ces philosophes pour qui la métaphysique reste une possibilité ouverte et vivante. Sa phénoménologie se démarque ainsi de la phénoménologie de Husserl dans la mesure où elle ne cherche pas seulement à analyser les phénomènes en tant que tels, mais encore à en tirer des conséquences «métaphysiques ». À cet égard, nous montrons que son projet peut s'inscrire dans une entreprise de réhabilitation de la métaphysique. Pour suivre cette intuition propre à Patočka, nous tentons de suivre un chemin qui nous conduit vers une nouvelle description de la phénoménalité. Ainsi, nous voulons montrer que le refus de toute ambition métaphysique empêche finalement de thématiser l'apparaître en tant que tel. Au contraire, 
l'interrogation métaphysique sur le concept de transcendance se manifeste comme la condition de l'accès à la question de l'apparaître. C'est dans ce cadre, celui d'un élargissement de la phénoménologie à la métaphysique, que nous avons poussé l'analyse jusqu'à ses limites, en abordant les thèmes de la promesse de l'expérience de la totalité et de la totalité qui apparaît comme une promesse. Car cette mise en avant de la figure de la promesse est ce qui doit nous permettre d'effectuer une synthèse, au niveau du sens, entre une description phénoménologique et une description métaphysique de la transcendance. 
Tonatiuh USECHE SANDOVAL

Directeur de thèse : Michel Bourdeau Laboratoire de rattachement : PhiCo Équipe NoSoPHi

\section{Les européocentristes sont-ils tous des colonialistes?}

Le post-colonialisme cherche à saper les racines spirituelles de la domination coloniale en s'attaquant à l'européocentrisme et à l'historicisme, qui posent l'Europe comme centre de l'humanité et l'histoire européenne comme préfiguration de l'histoire universelle. Grâce à la priorité spirituelle que leur accorde l'historicisme, les Européens peuvent asseoir leur prépondérance temporelle dans les relations internationales. Les positions de John Stuart Mill sur la présence européenne en Asie et en Afrique au $\mathrm{XIX}^{\mathrm{e}}$ siècle, ont servi à illustrer la thèse du post-colonialisme. Mill souligne le clivage entre les nations avancées, déjà civilisées et bien préparées pour la liberté, et les peuples arriérés, pas encore civilisés et bien disposés envers le despotisme. Le remplacement des despotismes indigènes par le despotisme civilisé des Européens apparaissait comme un facteur de civilisation pour les peuples barbares.

Malgré ses affinités avec Mill, la politique positive d'Auguste Comte, en conciliant un européisme actif et un anti-colonialisme sincère, constitue un contre-exemple à la thèse du post-colonialisme. Comte propose de refonder l'européocentrisme sur la reconstruction du lien européen. Ceci l'amène à envisager la colonisation comme triplement perturbatrice : elle détournait les Européens de la pressante question de refondre l'occidentalité ; elle fomentait des rivalités nationales en raison des guerres d'expansion à l'étranger; enfin, elle troublait le développement des populations moins avancées en y exportant l'anarchie occidentale. Comte est le tenant d'un européocentrisme relatif qui nuance le clivage entre civilisés et barbares, en soulignant tant les insuffisances des nations avancées que 
les mérites des peuples certes arriérés, mais aucunement attardés. Au nom de la vocation universelle de l'Europe, Comte a favorisé une décolonisation, et du colonisé et du colonisateur. 


\section{Élise SULTAN}

Laboratoire de rattachement : C.H.S.P.M.

Directeur de thèse : Jean Salem.

amsoline@hotmail.com

\section{Éros philosophe. \\ Romans libertins et philosophie au siècle des Lumières}

Si le siècle des Lumières est associé au siècle de la philosophie, des études récentes ${ }^{1}$ mettent en évidence l'importance concomitante de l'antiphilosophie dans la République des Lettres. En plus des griefs apologétiques, des réactions anti-Lumières animent des salons et des journaux, relayés par une littérature déclarant « la guerre à la philosophie $»^{2}$. Qu'en est-il de l'image de la philosophie et du personnage du philosophe dans les romans libertins du XVIII ${ }^{\mathrm{e}}$ siècle?

Classés parmi les «livres philosophiques» par les libraires, les romanciers libertins se jouent de cette collusion sous le manteau jusque dans leurs titres (Thérèse philosophe de Boyer d'Argens, La Philosophie dans le boudoir de Sade, etc., en attestent). Au-delà, un relevé des philosophèmes en présence montre des réminiscences épicuriennes ou des filiations avec Fréret, La Mettrie, Helvétius ou d'Holbach. Est-ce à dire que le roman du plaisir n'est qu'une coquetterie philosophico-érotique destinée à diffuser plus largement un discours préexistant dont il parerait l'amertume par le miel sulfureux de sa littérature ? Autrement dit, peut-on taxer le roman libertin de «philosophèmique » ou de «philosophique », lorsque le «libertinisme » désignerait un système de pensée singulier?

1. D. Masseau, Les ennemis des philosophes, Paris, Albin Michel, 2000 ; P. Hartmann et F. Lotterie (éds.), Le philosophe sur les planches et Le philosophe romanesque, Strasbourg, Presses Universitaires de Strasbourg, coll. Celus, 2004 et 2007.

2. C. Palissot, «Les philosophes », in O. Ferret (dir.), La comédie des Philosophes, SaintEtienne, Publications de l'Université de Saint-Etienne, 2002, Acte I, scène 1, vers 105, p. 29. 
Si ça philosophe en romancie libertine, la «philosophie», entre guillemets, est fustigée, non sans un certain humour, comme étant le discours creux des pédants dont la raison prétend triompher des plaisirs du corps, avec Platon pour parangon et ligne de mire. Peut-on pour autant parler d'antiphilosophie libertine ? Au-delà d'un effet de mode, que nous dit de la philosophie l'ironie libertine qui continue de sacrer philosophes ses plus grandes figures?

Les romans libertins proposent moins une réécriture littéraire de la philosophie qu'une nouvelle manière de philosopher dans le boudoir. Un art de vivre sous le signe d'Éros est mis en images par le phrasé libertin, telle une pulsation de plaisir, là où la philosophie des philosophes est sommée de tirer le rideau. 
Paola NICOLAS

Laboratoire de rattachement : Philosophie contemporaine Thèse dirigée par Denis Kambouchner pnicolas@ clipper.ens.fr

\section{Le témoignage du sentiment intérieur est-il fiable ? Analyses des controverses cartésiennes de Commercy}

Dom Robert Desgabets (1610-1678), un cartésien empiriste aux tendances aristotélisantes, accuse Descartes d'avoir angélisé nos âmes en supposant que nous puissions avoir des pensées indépendantes du corps. Celui-ci se situe dans le prolongement des Quatrièmes et Cinquièmes Objections en posant derechef une question tout à fait redoutable : sommesnous certains d'avoir dans le parcours des Méditations une perception complète de la res cogitans, ou plutôt comment être sûrs que nous ne rendions pas la connaissance de nous-mêmes inadéquate par une abstraction de l'esprit ? Desgabets récuse la possibilité de pouvoir faire abstraction de tout élément corporel lors de la seconde méditation. Parce que le cogito est une expérience proprement durative, celui-ci est la manifestation originaire de notre condition incarnée. Les objections robertistes s'élaborent à partir d'une sorte de «vide théorique » du discours cartésien : tout en s'efforçant de disqualifier le modèle de l'aevum pour penser la durée de notre âme, et en concevoir la radicale successivité, Descartes n'en formule pas clairement les conditions de possibilité. Profitant de cet « espace doctrinal », Desgabets construit sa critique en mettant en lumière le lien essentiel existant entre notre expérience d'une durée successive et notre épreuve de l'incarnation. 